\title{
Intrinsically tunable bulk acoustic wave resonators based on sol-gel grown PMN-PT films
}

\author{
A. Vorobiev, ${ }^{1, a)}$ M. Spreitzer, ${ }^{2}$ A. Veber, ${ }^{2}$ D. Suvorov, ${ }^{2}$ and S. Gevorgian ${ }^{1}$ \\ ${ }^{1}$ Department of Microtechnology and Nanoscience, Chalmers University of Technology, \\ SE-41296 Gothenburg, Sweden \\ ${ }^{2}$ Advanced Materials Department, Jožef Stefan Institute, SI-1000 Ljubljana, Slovenia
}

(Received 12 June 2014; accepted 4 August 2014; published online 13 August 2014)

\begin{abstract}
Intrinsically tunable bulk acoustic wave resonators, based on sol-gel $0.70 \mathrm{~Pb}\left(\mathrm{Mg}_{1 / 3} \mathrm{Nb}_{2 / 3}\right) \mathrm{O}_{3^{-}}$ $0.30 \mathrm{PbTiO}_{3}$ (PMN-PT) thin films, with high effective electromechanical coupling coefficient of $13 \%$ and tunability of the series resonance frequency up to $4.0 \%$ are fabricated and characterized. The enhanced electroacoustic properties of the PMN-PT resonators are attributed to the mechanism of polarization rotation occurring in the region of the morphotropic phase boundary. Electroacoustic performance of the PMN-PT resonators is analyzed using the theory of dc fieldinduced piezoelectric effect in ferroelectrics. Extrinsic acoustic loss in the PMN-PT resonators is analyzed using the model of the wave scattering at reflections from rough interfaces. Mechanical $Q$-factor of the resonators is up to 70 at $4.1 \mathrm{GHz}$ and limited mainly by losses in the PMN-PT film. (C) 2014 AIP Publishing LLC. [http://dx.doi.org/10.1063/1.4893179]
\end{abstract}

\section{INTRODUCTION}

The electrically tunable thin film bulk acoustic wave (BAW) resonators, utilizing electric field induced piezoelectric effect in paraelectric phase of ferroelectric materials have been intensively developed for the last five years. ${ }^{1-6}$ The $\mathrm{BAW}$ resonators based on the $\mathrm{BiFeO}_{3}-0.33 \mathrm{BaTiO}_{3}(\mathrm{BF}-\mathrm{BT})$ films with record high tunability of the resonance frequency of $4.4 \%$ and effective electromechanical coupling coefficient of $10 \%$ have been reported recently. ${ }^{7}$ However, for large scale industrial applications, for example, in the front ends of cellphones, personal communication systems, etc., even higher tunabilities and coupling coefficients are required. It is known that electromechanical response of the ferroelectric materials is exceptionally high near the morphotropic phase boundary (MPB), which is a region on the phase diagram separating phases with different symmetries. ${ }^{8}$ In particular, solid solutions of the relaxor-ferroelectrics $(1-x) \mathrm{Pb}\left(\mathrm{Mg}_{1 / 3} \mathrm{Nb}_{2 / 3}\right)$ $\mathrm{O}_{3}-x \mathrm{PbTiO}_{3}$, usually abbreviated as PMN- $x \mathrm{PT}$, show a superior electromechanical performance across the MPB. ${ }^{8,9}$ On the PMN- $x$ PT phase diagram, at room temperature, the MPB is defined by a narrow, $x \approx 0.30-0.34$, monoclinic phase region separating the rhombohedral and tetragonal phases. ${ }^{9}$ The enhancement of intrinsic piezoelectric activity of the PMN- $x$ PT ferroelectrics with $x \leq x_{\mathrm{MPB}}$ is attributed to the rotation of the polarization from the $[111]_{\text {cub }}$ to $[001]_{\text {cub }}$ directions, which are directions of the cation displacements in the rhombohedral and tetragonal phases, respectively, induced by an electric field applied along [001] $]_{\text {cub }}$ direction. ${ }^{8,10}$ This mechanism of the polarization rotation is responsible for that the field induced piezoelectric coefficient, $d_{33}$, in the $[001]_{\text {cub }}$ oriented PMN-xPT crystals around the $\mathrm{MPB}$, can be as high as $3000 \mathrm{pm} / \mathrm{V} .{ }^{11}$ Additionally, the [001] oriented PMN- $x \mathrm{PT}$ single crystals show maximum of the

\footnotetext{
a) Author to whom correspondence should be addressed. Electronic mail: andrei.vorobiev@chalmers.se
}

coupling factor in the MPB region, $x \approx 0.30-0.34$, as high as $62 \% .{ }^{12}$ Studies of the PMN- $x$ PT ceramics in the composition range $x=0.0-0.4$ show clear maximum of the $d_{33}$ up to $1000 \mathrm{pm} / \mathrm{V}$ at $x \approx 0.30$, which confirms the MPB effect. ${ }^{13}$ For comparison, simple calculations based on the results of the direct piezoelectric effect measurements, indicate that the $\mathrm{Mn}$ modified $\mathrm{BiFeO}_{3}-0.33 \mathrm{BaTiO}_{3}$ ceramic possesses significantly lower $d_{33} \approx 14 \mathrm{pm} / \mathrm{V} .{ }^{14}$ Therefore, one can expect that the BAW resonators utilizing the [001] oriented PMN- $x$ PT ferroelectric with composition close to the MPB should possess intrinsically high tunability of the resonance frequency and electromechanical coupling coefficient. However, there are only a few reports on use of the PMN- $x$ PT ferroelectrics in the BAW resonators, mainly as single crystals, and, hence, at relatively low frequencies. In particular, a PMN- $x$ PT single crystal resonator, operating at $20 \mathrm{MHz}$, is demonstrated for highly sensitive vapor detection and proposed for applications in advanced biochemical sensors. ${ }^{15}$ Integration of a PMN- $x$ PT single crystal resonator in a microfluidic system, operating in shear mode, in the frequency range $2-20 \mathrm{MHz}$, is reported and proposed for dynamic monitoring of fluid properties. ${ }^{16}$ Electroacoustic properties of single-domain PMN-0.33PT thin films are studied at resonance frequency of 1.3 GHz and high potential of the PMN-PT based BAW transducers is indicated. ${ }^{17}$ To the best of our knowledge, there are no reports on the BAW resonators utilizing the PMN- $x$ PT thin films. In this paper, we report performance of the BAW resonators fabricated using the [001] textured PMN-0.30PT thin films grown by sol-gel technique.

\section{EXPERIMENTAL DETAILS}

The PMN-0.30PT (PMN-PT) BAW resonator test structures are fabricated as solidly mounted devices on silicon substrates with resistivity of $20 \mathrm{k} \Omega \cdot \mathrm{cm}$. The Bragg reflector, consisting of $\lambda / 4 \mathrm{SiO}_{2} / \mathrm{W}$ layers with thicknesses of 210 / $180 \mathrm{~nm}, 50 / 10 \mathrm{~nm}$ thick $\mathrm{TiO}_{2} / \mathrm{Ti}$ diffusion barrier/adhesion 
layers and $80 \mathrm{~nm}$ thick Pt bottom plate are deposited by magnetron sputtering. The PMN-PT films, with three different thicknesses of 270, 360, and $440 \mathrm{~nm}$, are grown on the $\mathrm{Pt}$ bottom plate using a sol-gel process. The sols are prepared using the $\mathrm{Mg}\left(\mathrm{NO}_{3}\right)_{2} \cdot 6 \mathrm{H}_{2} \mathrm{O}, \mathrm{Nb}\left(\mathrm{OC}_{2} \mathrm{H}_{5}\right)_{5}, \mathrm{Ti}\left(\mathrm{C}_{4} \mathrm{H}_{9}\right)_{4}$ and $\mathrm{Pb}\left(\mathrm{NO}_{3}\right)_{3} \quad 2 \mathrm{H}_{2} \mathrm{O}$ as the precursors, 2-ethoxyethanol as the solvent and acetylacetone $\mathrm{C}_{5} \mathrm{H}_{8} \mathrm{O}_{2}$ as the complex additive. Polyvinylpyrrolidione (average mass weight is 58000) is used to control the reactivity of $\mathrm{Pb}$ ions toward $\mathrm{Nb}$ and, consequently, the formation of a pyrochlore phase. The thin films are deposited using the spin-coating method at $3000 \mathrm{rpm}$ for $30 \mathrm{~s}$, preheated and heat treated at temperature of $450^{\circ} \mathrm{C}$ for $10 \mathrm{~min}$ and $650^{\circ} \mathrm{C}$ for $20 \mathrm{~min}$, respectively. For the study synthesis conditions were optimized to reduce the pyrochlore concentration and increase the films texturing. The 20/90/10 nm thick Au/Al/Ti top electrode stack is deposited by e-beam evaporation. The top Au film is used to minimize the contact resistance between the contact pads and the microprobe tips. A lift-off process is used to pattern the top electrodes in the form of central circular electrodes surrounded by concentric outer electrodes with inner diameter of $200 \mu \mathrm{m}$. The BAW resonator test structures with the top electrodes of 40,60 , and $80 \mu \mathrm{m}$ in diameter are fabricated and characterized. In the analysis below, the resonators with $40 \mu \mathrm{m}$ top electrode are used, if not indicated specifically. The schematic cross section view and layout of the BAW resonator test structures are shown in Fig. 1. The dc and microwave connection between the microprobe tips and the Pt bottom electrode is achieved through the PMN-PT film by means of the resistive/capacitive divider. The relatively large area of the outer electrode provides effective dc and microwave connection to the Pt bottom electrode.

The microstructure of the PMN-PT films is analyzed using X-ray diffraction (XRD) and scanning electron microscopy (SEM) techniques. The XRD spectra are obtained using a PANalytical Empyrean system with a hybrid monochromator, consisting of a combination of an X-ray mirror and a two-crystal Ge(220) two-bounce monochromator. The spectra were acquired in $\omega-2 \theta$ mode in the range from $20^{\circ}$ to $50^{\circ}$ with $0.013^{\circ}$ step size and $300 \mathrm{~s}$ per step. The SEM images of the PMN-PT film are obtained using a Carl Zeiss Ultra Plus SEM.

The complex input impedance $Z=\operatorname{Re} Z+\mathrm{j} \operatorname{Im} Z$ and admittance of the test structures are calculated using $S_{11}$

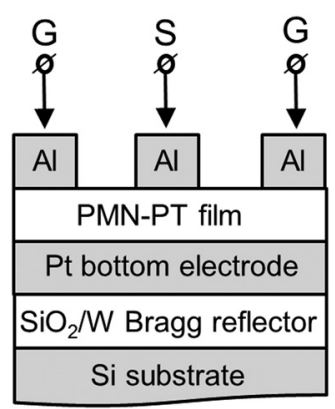

(a)

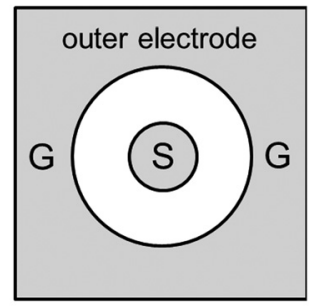

(b)
FIG. 1. Schematic cross section view (a) and layout (b) of PMN-PT BAW resonators. Signal and ground contacts of the microprobe and corresponding contact pads are labeled as "S" and "G," respectively. parameters measured using an Agilent N5230A vector network analyzer and ground-signal-ground microprobes, see Fig. 1, in the frequency range $1-10 \mathrm{GHz}$. The modified Butterworth-Van Dyke (mBVD) circuit model is used for de-embedding the electrical loss associated with the series resistance $R_{\mathrm{S}}$ composed, mainly, by the ring section of the $\mathrm{Pt}$ bottom plate. The series resistance in the mBVD model is found as the real part of impedance in the high frequency limit. ${ }^{18}$ The series $\left(f_{\mathrm{s}}\right)$ and parallel $\left(f_{\mathrm{p}}\right)$ resonance frequencies are determined at the maximum of the real parts of the complex admittance and impedance, respectively.

\section{RESULTS AND DISCUSSION}

\section{A. Microstructure}

Fig. 2 shows the XRD pattern of a PMN-PT film grown on a platinized $\mathrm{Si}$ substrate, which is nearly phase-pure perovskite with only a trace of the pyrochlore phase. Peak indexing is done on the basis of the pseudocubic unit cell. The comparison with the XRD pattern of the ceramic counterpart (Ref. 19) indicates that the PMN-PT film is (001) textured. The texturing ratio is defined as: ${ }^{20}$

$$
M=\frac{I_{(001)}^{\mathrm{f}}}{I_{(001)}^{\mathrm{c}}} \cdot \frac{I_{(001)}^{\mathrm{c}}+I_{(110)}^{\mathrm{c}}+I_{(111)}^{\mathrm{c}}}{I_{(001)}^{\mathrm{f}}+I_{(110)}^{\mathrm{f}}+I_{(111)}^{\mathrm{f}}},
$$

where $I_{(\mathrm{hkl})}^{\mathrm{f}}$ and $I_{(\mathrm{hkl})}^{\mathrm{c}}$ are the integrated intensities of the (hkl) peaks of the film and ceramic, respectively. Calculations using Eq. (1) give texturing ratio of 8.6. Since composition of the PN-PT films is at MPB, one can expect high piezoelectric and electrostrictive properties due to the mechanism of polarization rotation and, consequently, the high tunability and coupling coefficient of the BAW resonators. ${ }^{8,10}$

Fig. 3 shows SEM top and cross section images of a $270 \mathrm{~nm}$ thick PMN-PT film. It can be seen that, to the large extend, the grains are columnar with lateral grain size of $100-250 \mathrm{~nm}$.

The columnar grain structure implies that the film effective capacitance, and permittivity, can be described in terms of capacitances associated with the grains and grain boundaries connected in parallel. In this case contribution of the grain boundaries to the film effective permittivity and its tunability, $n_{\mathrm{r}}$, is negligible. Therefore, the electroacoustic

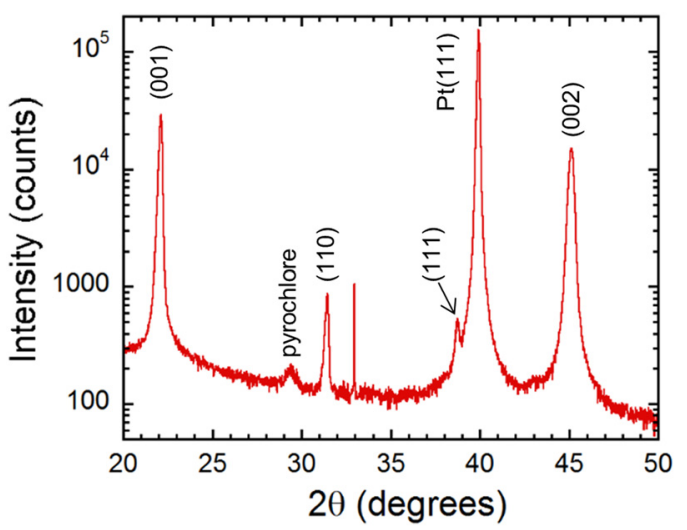

FIG. 2. XRD pattern of a PMN-PT film grown on a platinized Si substrate. 

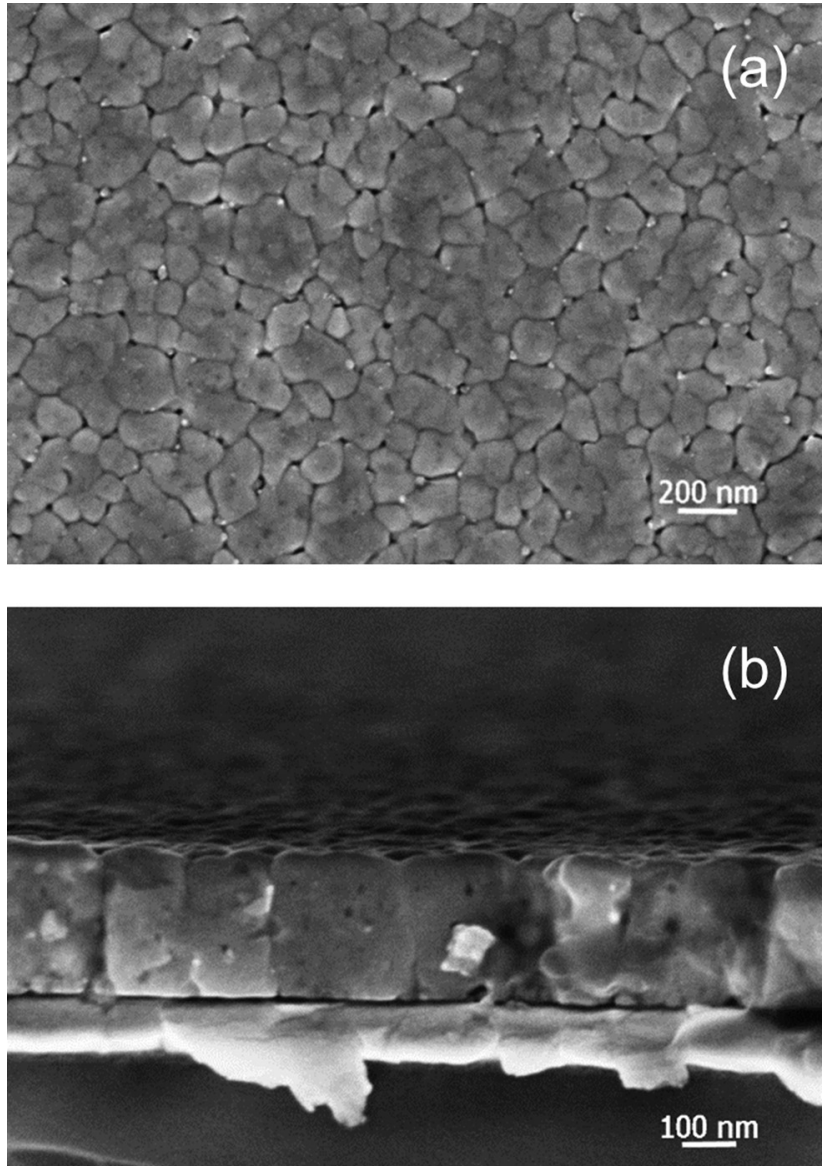

FIG. 3. SEM top (a) and cross section (b) images of a $270 \mathrm{~nm}$ thick PMNPT film.

performance of the BAW resonators is governed mainly by the grain properties of the PMN-PT film, see Eqs. (3)-(5). It can be seen from Fig. 3 that the top surface of the PMN-PT film is significantly rougher than the bottom interface. This implies that the extrinsic acoustic loss associated with the acoustic wave scattering at reflection from a rough interface is defined mainly by the top surface of the PMN-PT BAW resonator. More detailed analysis of this loss mechanism is given in the next section.

\section{B. Resonator performance}

Fig. 4 shows conductance, i.e., real part of admittance, normalized to $50 \Omega$, of the BAW resonators with $270 \mathrm{~nm}$, $360 \mathrm{~nm}$, and $440 \mathrm{~nm}$ thick PMN-PT films versus frequency at the same $35 \mathrm{~V} / \mu \mathrm{m}$ strength of the applied dc electric field. Shown also are the reflection coefficients of the Bragg reflector calculated for the longitudinal and shear acoustic waves incident from an infinitely thick PMN-PT layer. It can be seen from Fig. 4 that magnitude of the conductance peaks, in the frequency range $3-4 \mathrm{GHz}$, decreases rapidly with increase in the PMN-PT film thickness. The drop in the conductance clearly correlates with the low frequency edge of the longitudinal wave reflection band. Therefore, one can conclude that performance of the BAW resonators with thicker PMN-PT films, and, correspondingly, lower resonance frequencies, degrades due to more intensive leak of the acoustic waves through the Bragg reflector. A fluffy

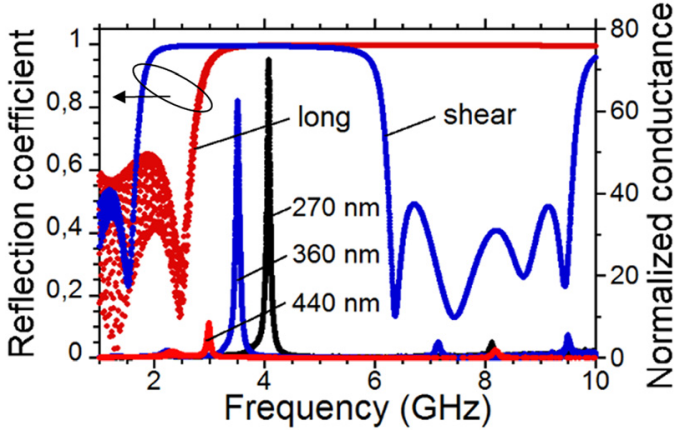

FIG. 4. Calculated reflection coefficients of the longitudinal and shear waves and measured normalized conductance of the BAW resonators with $270 \mathrm{~nm}$, $360 \mathrm{~nm}$, and $440 \mathrm{~nm}$ thick PMN-PT films vs. frequency at $35 \mathrm{~V} / \mu \mathrm{m}$ dc electric field.

structure of the $440 \mathrm{~nm}$ conductance peak formed by multiple reflections from the back side of the Si substrate confirms this conclusion. Another important conclusion is that the resonances are associated with longitudinal waves since the low frequency edge of the shear wave reflection band is at significantly lower frequency. Due to the substantial leak of the acoustic waves through the Bragg reflector the BAW resonators with $440 \mathrm{~nm}$ thick PMN-PT films are not considered in the further analysis.

Fig. 5 shows dc bias voltage dependences of the permittivity of the $270 \mathrm{~nm}$ thick PMN-PT films for one cycle of the voltage sweep, measured at $1 \mathrm{MHz}$ and at the resonance frequency of $4.1 \mathrm{GHz}$. It can be seen that there is a frequency dispersion of the permittivity. The permittivity at zero dc field is reduced from, approximately, 650 down to 500 at $1 \mathrm{MHz}$ and $4.1 \mathrm{GHz}$, respectively. Besides, the permittivity dependences reveal, approximately, $10 \%$ hysteresis at both frequencies. This behavior can be attributed to the contribution of irreversible polarization of polar nanoclasters with distributed relaxation frequency. ${ }^{8}$ Fig. 5 shows also relative tunability of permittivity calculated as

$$
n_{\mathrm{r}}=\frac{\varepsilon(0)-\varepsilon(E)}{\varepsilon(0)},
$$

where $\varepsilon(0)$ and $\varepsilon(E)$ are the permittivity without and with dc electric field, respectively. The relative tunability is,

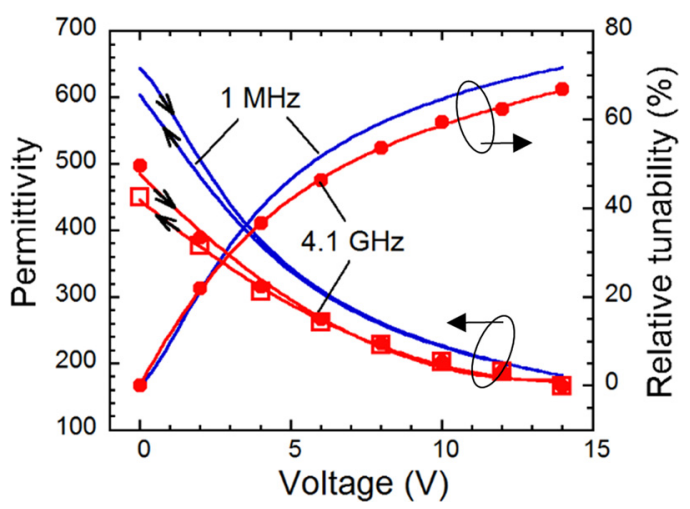

FIG. 5. Permittivity and relative tunability of the permittivity of the $270 \mathrm{~nm}$ thick PMN-PT films vs. applied dc voltage at $1 \mathrm{MHz}$ and at a resonance frequency of $4.1 \mathrm{GHz}$. Permittivity dependences are shown for one cycle of dc voltage sweep varying according to arrow directions. 


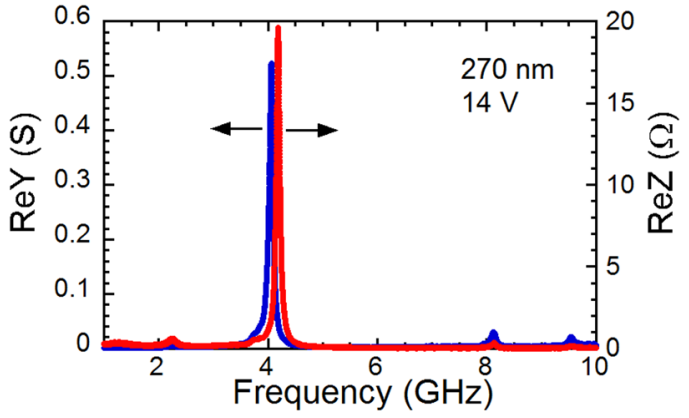

FIG. 6. Real parts of admittance (ReY) and impedance (ReZ) of the BAW resonator with $270 \mathrm{~nm}$ PMN-PT film vs. frequency at $14 \mathrm{~V}$.

approximately, $72 \%$ and $67 \%$ at $1 \mathrm{MHz}$ and $4.1 \mathrm{GHz}$, respectively. Reduced tunability at $4.1 \mathrm{GHz}$ can be explained by the frequency dispersion of the permittivity.

Fig. 6 shows the real parts of admittance and impedance of the BAW resonator with $270 \mathrm{~nm}$ PMN-PT film versus frequency at $14 \mathrm{~V}$. The maximums of the dependences determine the series and parallel resonance frequencies, respectively.

Fig. 7 shows the series and parallel resonance frequencies of the BAW resonators with $270 \mathrm{~nm}$ and $360 \mathrm{~nm}$ PMNPT films versus electric field of the applied dc bias. The series resonance frequencies of the $270 \mathrm{~nm}$ BAW resonator are shown for the one cycle of the dc voltage sweep. It can be seen that the hysteresis of the series resonance frequency is below $0.2 \%$, which is much less than the resonance frequency tunability and can be ignored for practical applications. The series resonance frequency decreases while the parallel resonance frequency increases with field for both PMN-PT film thicknesses. The parallel resonance frequency reveals much weaker field dependence than that of the series resonance. The highest applied electric field corresponds to the breakdown field. As it can be seen from Fig. 7, the breakdown field strength is lower for the thinner film, which can be explained by an effect of an interfacial layer with reduced breakdown field.

Fig. 8 shows tunabilities of the series $\left(n_{\mathrm{s}}\right)$ and parallel $\left(n_{\mathrm{p}}\right)$ resonance frequencies of the $\mathrm{BAW}$ resonators with $270 \mathrm{~nm}$ and $360 \mathrm{~nm}$ PMN-PT films versus electric field of the applied dc bias. The tunability is defined as

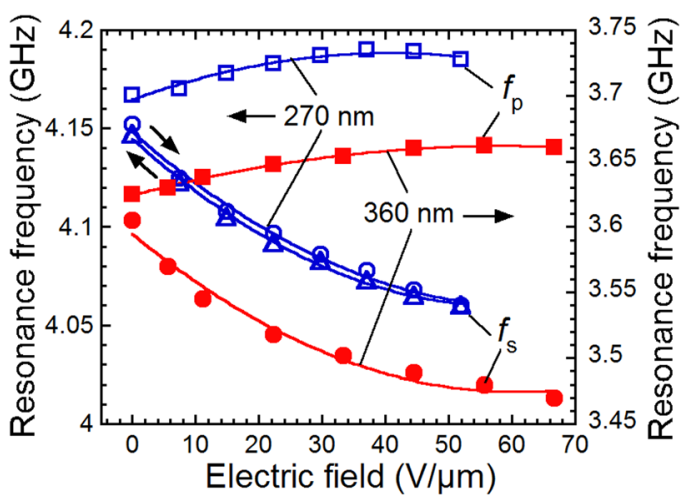

FIG. 7. Series $\left(f_{\mathrm{s}}\right)$ and parallel $\left(f_{\mathrm{p}}\right)$ resonance frequencies of the BAW resonators with $270 \mathrm{~nm}$ and $360 \mathrm{~nm}$ PMN-PT films vs. applied dc electric field. The series resonance frequencies of the $270 \mathrm{~nm}$ BAW resonator is shown for one cycle of the dc voltage sweep varying according to arrow directions.

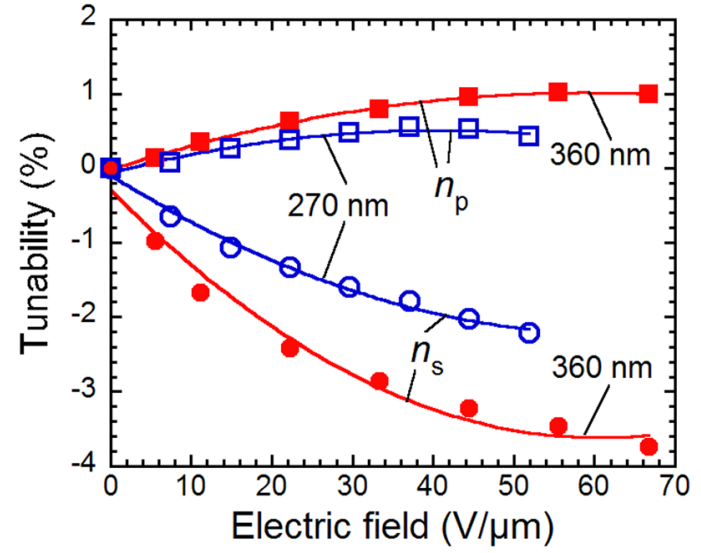

FIG. 8. Tunabilities of the series $\left(n_{\mathrm{s}}\right)$ and parallel $\left(n_{\mathrm{p}}\right)$ resonance frequencies of the BAW resonators with $270 \mathrm{~nm}$ and $360 \mathrm{~nm}$ PMN-PT films vs. applied dc electric field.

$$
n_{\mathrm{s}, \mathrm{p}}=\frac{f_{\mathrm{s, \textrm {p }}}-f_{0}}{f_{0}},
$$

where $f_{0}$ is the resonance frequency extrapolated to zero dc electric field. The electroacoustic performance of the PMNPT film BAW resonators can be analyzed using the theory of the dc field induced piezoelectric effect. ${ }^{1}$ This theory established following relation between the electromechanical coupling factor $k_{\mathrm{f}}^{2}$ of a non-loaded ferroelectric film and its relative tunability of permittivity: ${ }^{1}$

$$
k_{\mathrm{f}}^{2} \approx \frac{4 q^{2}}{3 c^{0} \beta} n_{\mathrm{r}}=A_{\mathrm{t}} n_{\mathrm{r}},
$$

where $q, \beta$, and $c^{0}$ are corresponding components of the tensors of linear electrostriction, dielectric non-linearity and elastic constant at zero dc electric field, respectively. The dc bias dependent tunabilities of the series $\left(n_{\mathrm{sf}}\right)$ and parallel $\left(n_{\mathrm{pf}}\right)$ resonance frequencies may be described in terms of $k_{\mathrm{f}}^{2}$ (or $n_{\mathrm{r}}$, see Eq. (4)) as:

$$
\begin{gathered}
n_{\mathrm{sf}}=-k_{\mathrm{f}}^{2}\left(\gamma+\frac{\mu}{2}+\frac{4}{\pi^{2}}\right), \\
n_{\mathrm{pf}}=-k_{\mathrm{f}}^{2}\left(\gamma+\frac{\mu}{2}\right),
\end{gathered}
$$

where

$$
\begin{gathered}
\gamma \approx \frac{m}{8 q^{2} \varepsilon \varepsilon_{0}}, \\
\mu \approx \frac{\varepsilon^{b}}{\varepsilon},
\end{gathered}
$$

$m$ and $\varepsilon^{\mathrm{b}} \approx 7$ are the corresponding components of the tensors of non-linear electrostriction and the background permittivity, respectively. ${ }^{21}$ Typically, both parameters $\gamma$ and $\mu$ are of the same order of magnitude and much smaller than unit. This indicates that $4 / \pi^{2}$ is clearly the leading term in brackets of Eq. (5), which results in stronger field dependence and, correspondingly, higher tunability of the series resonance frequency in comparison with the parallel resonance 
frequency (Figs. 7 and 8). The positive tunability of the parallel resonance frequency (Figs. 7 and 8) is due to negative sign of the sum of $\gamma+\mu / 2$, see Eq. (6). It was shown that the sign of the $\gamma+\mu / 2$, is not controlled by any general reason and may differ between materials depending on the relationship between $\gamma$ and $\mu^{22}$ The most of demonstrated previously BAW resonators, based on the $\mathrm{Ba}_{\mathrm{x}} \mathrm{Sr}_{1-\mathrm{x}} \mathrm{TiO}_{3}$ (BSTO) films with $x<0.5$, reveal negative tunability of the parallel resonance frequency, i.e., softening. ${ }^{1,4,18,23-26}$ Recent $a b$ initio calculations indicate that the non-linear electrostrictive coefficient is negative in the whole range of the BSTO composition. ${ }^{27}$ It means that the term $\mu / 2$ dominates in the sum of $\gamma+\mu / 2$ with $x<0.5$. However, the BSTO BAW resonators with $x \geq 0.5$ reveal positive tunability of the parallel resonance frequency, i.e., stiffening. ${ }^{28,29}$ This can be explained by lower $\mu$ value due to higher permittivity of the material with higher Ba concentration and, hence, domination of the term $\gamma$ in the sum of $\gamma+\mu / 2$. For the PMN-PT, no published data on the non-linear electrostrictive coefficients are available to the authors' knowledge, which does not allow for correct analysis of the relationship between $\gamma$ and $\mu$. We suppose that the observed positive tunability of the parallel resonance frequency (Figs. 7 and 8) can also be explained by lower $\mu$ value since the PMN-PT permittivity is rather high (Fig. 5).

As it can be seen from Fig. 8, tunabilities of the resonance frequencies of the BAW resonators with $270 \mathrm{~nm}$ thick PMN-PT film is lower than those with $360 \mathrm{~nm}$ thick film. This is due to the effect of loading by electrodes. The mechanical loading of the piezoelectric film by the electrodes results in the loss of acoustic energy in the non-piezoelectric electrode layers and, consequently, reduction of the BAW resonator tunability and effective electromechanical coupling coefficient, in comparison with the intrinsic film parameters. ${ }^{23,30}$ However, the tunability of the series resonance frequency of the BAW resonator with $360 \mathrm{~nm}$ thick PMN-PT film is up to $4 \%$, which is very close to the highest reported $4.4 \%$ of the BAW resonators based on the BF-BT films. ${ }^{7}$

Fig. 9 shows effective electromechanical coupling coefficients of the BAW resonators with $270 \mathrm{~nm}$ and $360 \mathrm{~nm}$ PMN-PT films versus electric field of the applied dc bias. The effective electromechanical coupling coefficient is calculated as

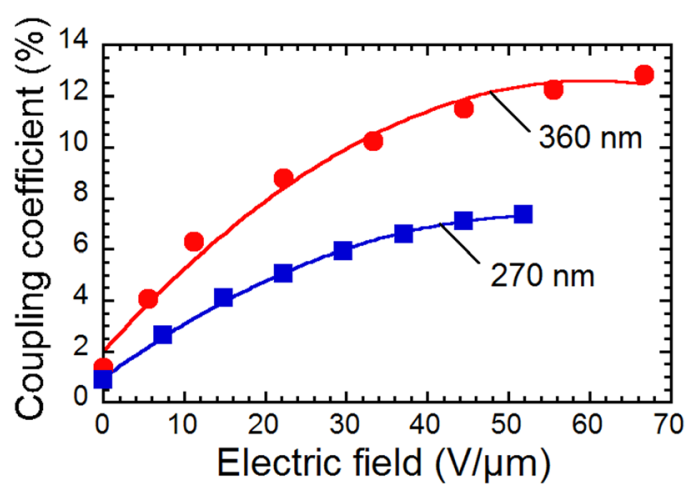

FIG. 9. Effective electromechanical coupling coefficients of the BAW resonators with $270 \mathrm{~nm}$ and $360 \mathrm{~nm}$ PMN-PT films vs. applied dc electric field.

$$
k_{\mathrm{eff}}^{2}=\frac{\pi^{2}}{4} \frac{f_{\mathrm{p}}-f_{\mathrm{s}}}{f_{\mathrm{p}}} .
$$

The coupling coefficient of the resonator with $360 \mathrm{~nm}$ thick film is up to $13 \%$, which is among the highest of those reported for the intrinsically tunable BAW resonators. ${ }^{5}$ The coupling coefficient of the BAW resonators with $270 \mathrm{~nm}$ PMN-PT film is lower than that with $360 \mathrm{~nm}$ due to the effect of loading by the electrodes.

The tunabilities and effective electromechanical coupling coefficient of the loaded BAW resonator follow the same dependences on the dc field, as in the non-loaded case, remaining the linear functions of the relative tunability of permittivity, as in Eqs. (4)-(6). ${ }^{23}$ Subtracting Eqs. (5) and (6) gives tunability difference

$$
n_{\mathrm{sf}}-n_{\mathrm{pf}}=-\frac{4}{\pi^{2}} k_{\mathrm{f}}^{2},
$$

which is valid also for the loaded BAW resonator parameters

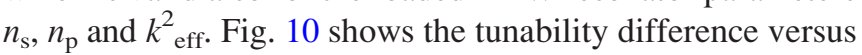
effective electromechanical coupling coefficient of the BAW resonators for both PMN-PT film thicknesses, $270 \mathrm{~nm}$ and $360 \mathrm{~nm}$. It can be seen that the dependences are practically identical, linear with the slope of, approximately, -0.40 . This is very close to the $-4 / \pi^{2} \approx-0.405$ predicted by theory for the non-loaded piezoelectric layer, see Eq. (10), which confirms the validity of the theory in the case of the PMNPT films.

Fig. 10 shows also the effective electromechanical coupling coefficients of the BAW resonators plotted versus relative tunability of permittivity. It can be seen that the dependences can be approximated by linear functions, which is in agreement with the theory, see Eq. (4). Some nonlinearity appearing at the highest values of the relative tunability of permittivity can be explained by the fact that Eq. (4) is strictly valid in the limit of small tuning, i.e., small fields. The slope of dependences allows for estimation of the proportionality factor $A_{\mathrm{t}}$, which characterizes the tunable and electromechanical coupling performance of the BAW resonators. For the $360 \mathrm{~nm}$ thick PMN-PT film $A_{\mathrm{t}} \approx 0.15$. For

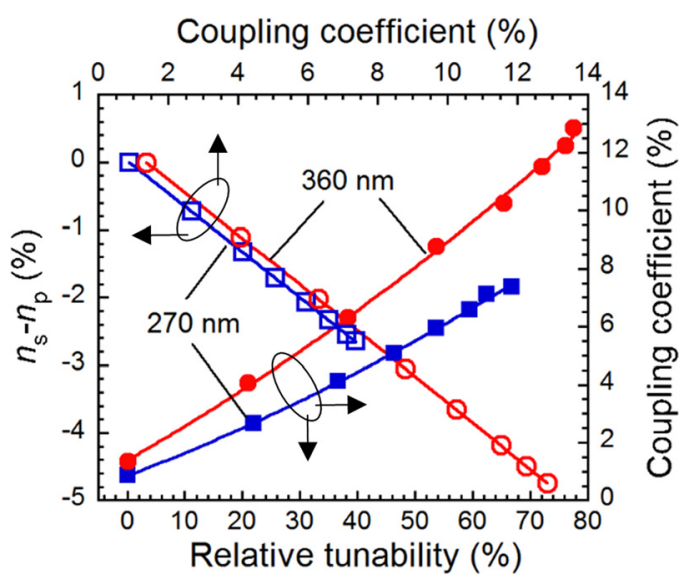

FIG. 10. Tunability difference $n_{\mathrm{s}}-n_{\mathrm{p}}$ vs. effective electromechanical coupling coefficient and the coupling coefficient vs. relative tunability of permittivity of the BAW resonators with $270 \mathrm{~nm}$ and $360 \mathrm{~nm}$ PMN-PT films. 
comparison, the BF-BT BAW resonators with the similar electrodes and, approximately, same ferroelectric film thickness of $330 \mathrm{~nm}$ reveal more than two times larger $A_{\mathrm{t}} \approx 0.40 .^{7}$ Similarity of the resonance frequency tunabilities and even higher effective electromechanical coupling coefficient of the PMN-PT BAW resonators can be explained by the significantly higher relative tunability of permittivity, $80 \%$ versus $20 \%$ for the BF-BT films. ${ }^{7}$

Fig. 11 shows quality factors of the BAW resonators with $270 \mathrm{~nm}$ and $360 \mathrm{~nm}$ PMN-PT films versus applied dc voltage for different diameters of the top electrode. The $Q$ factor is calculated as

$$
Q=\left.\frac{1}{2} f_{\mathrm{s}} \frac{\partial \varphi_{\mathrm{de}}}{\partial f}\right|_{f=f_{\mathrm{s}}},
$$

where

$$
\varphi_{\mathrm{de}}=\operatorname{arctg} \frac{\operatorname{Im} Z}{\operatorname{Re} Z-R_{\mathrm{s}}}
$$

is the de-embedded phase angle. The de-embedding of the electrical loss allows for considering the $Q$-factor calculated using Eq. (11) as the pure mechanical one $\left(Q_{\mathrm{m}}\right)$. It can be seen from Fig. 11 that dependences clearly reveal maximums, which is explained by the interplay between the bias dependencies of the motional inductance and resistance. ${ }^{31}$ Besides, it can be seen that the $Q$-factors of the BAW resonators with the larger top electrode area are significantly larger. We assume that the effects associated with deformations, scratches and mechanical loading of the resonator top electrode by the probe tip are minor. According to our observations the probe contact area is less than $30 \mu \mathrm{m}^{2}$, which is
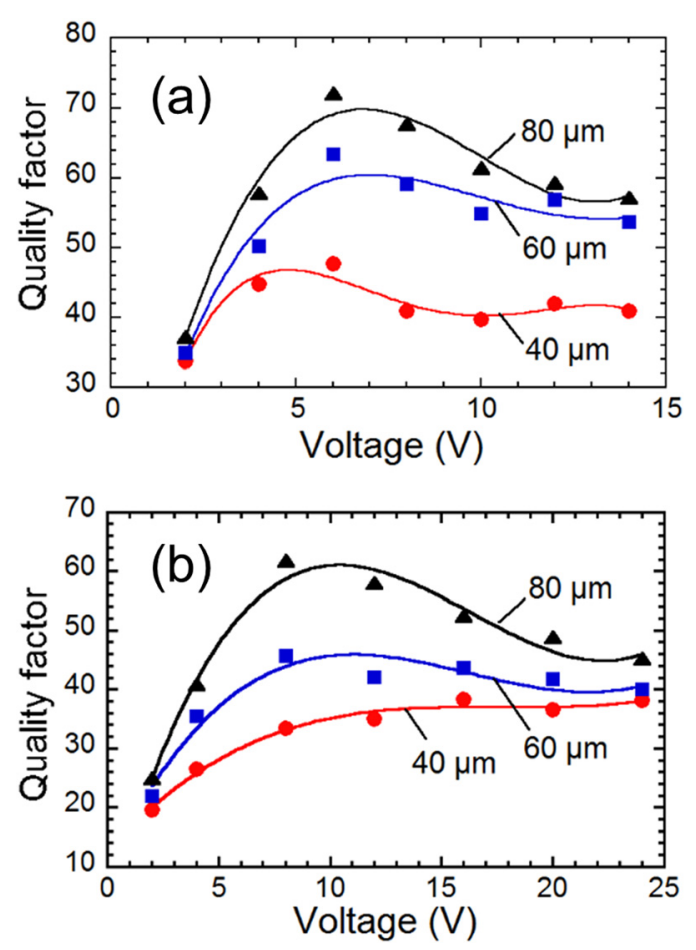

FIG. 11. The quality factors of the BAW resonators with $270 \mathrm{~nm}$ (a) and $360 \mathrm{~nm}$ (b) PMN-PT films vs. applied dc voltage for different diameters of the top electrode. much less than the smallest used top electrode area $1256 \mu \mathrm{m}^{2}$. Our previous measurements of the BSTO BAW resonators with the top electrode area varying in the range of $300-3000 \mu \mathrm{m}^{2}$ reveal rather weak dependence of the $Q$-factor on the area, $5.5 \%$ in the range of $1200-3000 \mu \mathrm{m}^{2}$ on the level of $Q \approx 350 .^{6}$ Finally, detailed comparison with the BAW resonators having external contact pads reveal similarity of the $Q$-factors within $5 \%$, which is comparable with accuracy of the $Q$-factor evaluation. ${ }^{32}$ The size dependence observed for the PMN-PT BAW resonators, Fig. 11, can be explained assuming a loss mechanism associated with the edges of the top electrode. For example, fringing field at the edges can induce an inclined polarization generating the acoustic shear waves. If the shear waves leak through the Bragg reflector, they consequently lower the $Q$-values. The shear waves are not necessarily to be at resonance conditions. It was shown that the loss can be significant for even small amounts of energy associated with the shear waves. ${ }^{33}$ The resonant peaks appeared at, approximately, $7.2 \mathrm{GHz}$ and $8.2 \mathrm{GHz}$ in the BAW resonators with $270 \mathrm{~nm}$ and $360 \mathrm{~nm}$ thick PMN-PT films, respectively, (see Fig. 4) can be associated with the shear waves. As it can be seen from Fig. 4, they are outside of the main shear wave reflection band. More detailed analysis has shown that these peaks reveal strongly irreversible behavior indicating their remnant polarization origin. Therefore, they cannot be attributed to the second harmonics of the main longitudinal waves associated with the reversible polarization. It can be seen from Fig. 11 that the $Q$-factors of the BAW resonators with the $360 \mathrm{~nm}$ thick PMN-PT films are slightly lower than those with $270 \mathrm{~nm}$ thick films. Most likely, this is because of more intensive wave leaking through the Bragg reflector since the corresponding resonant frequency is closer to the edge of the longitudinal wave reflection band (see Fig. 4).

It was shown that in the ferroelectric BAW resonators the main extrinsic loss mechanism is associated with wave scattering at reflections from rough interfaces, which assumes redirection of vertically moving acoustic energy toward lateral directions. ${ }^{34}$ This causes the waves to leave the active resonator region and dissipate either in the device substrate or in the region surrounding the device laterally. ${ }^{35} \mathrm{It}$ can be seen from Fig. 3, that the $\mathrm{Pt} / \mathrm{SiO}_{2}$ interface, which is the main bottom reflecting surface, is relatively smooth. Therefore, we assume that the scattering loss is mainly defined by the top electrode roughness which is approximately the same as that of the PMN-PT film surface (Fig. 3). Scattering takes place mainly once per cycle at reflection from the top electrode surface. It can be seen from Fig. 3 that the transverse roughness scale is defined by the lateral grain size, which is $100-250 \mathrm{~nm}$. The transverse roughness scale is much smaller than the wavelength, which is, approximately, $1000 \mathrm{~nm}$. At a single reflection, when the transverse roughness scale is much smaller than the wavelength, the attenuation coefficient can be approximated in $\mathrm{dB}$ as: ${ }^{36}$

$$
\alpha_{\mathrm{dB}}=2 \pi 8.68 k^{2} \eta^{2} \mathrm{~dB}
$$

where $k=2 \pi / \lambda$ is the longitudinal wave number, $\lambda$ is the wavelength and $\eta$ is the rms surface roughness. Using a 
general definition of the $Q$-factor as the ratio between energy stored and energy dissipated per cycle, the $Q$-factor of the BAW resonator, associated with wave scattering at top interface only, can be calculated as:

$$
Q_{\mathrm{sc}}=2 \pi \frac{E_{\mathrm{tot}}}{E_{\mathrm{tot}}-E_{\mathrm{ref}}}=\frac{2 \pi}{1-\frac{1}{\alpha}},
$$

where $E_{\text {tot }}$ and $E_{\text {ref }}$ represent the energies of incoming and reflected acoustic waves, respectively, $\alpha=E_{\text {tot }} / E_{\text {ref }}$ is the attenuation coefficient and $\log \alpha=0.1 \alpha_{\mathrm{dB}}$. Assuming that the wavelength equals to the double thickness of the ferroelectric film and electrodes for the $270 \mathrm{~nm}$ thick PMN-PT film one can readily get $\lambda=940 \mathrm{~nm}$. The rms surface roughness of the PMN-PT film is estimated analyzing the SEM cross section image, Fig. 3 , with an error of, approximately, $\pm 5 \%$. This is relatively less accurate method than the atomic force microscopy, which we used before. ${ }^{6,7,18,26,28}$ However, this accuracy is sufficient for our analysis below demonstrating relationship between the $Q$-factors associated with the scattering loss and overall losses within the PMN-PT film. The error in the $Q$-factor evaluation is less than $\pm 9 \%$. The advantage and reason of using the SEM cross section image are that it allows for direct association of the surface morphology with the film grain structure, see Fig. 3. Calculations using profile heights at 21 points over the whole cross section area give $\eta=7.19 \mathrm{~nm}$. Then, using Eqs. (13) and (14), one can get the $Q$-factor associated with loss due to scattering at reflection from the top electrode of the PMN-PT BAW resonator as $Q_{\mathrm{sc}}=220$.

The overall mechanical $Q$-factor of the BAW resonator can be expressed as: ${ }^{33}$

$$
Q_{\mathrm{m}}=\left(\frac{1}{Q_{\mathrm{f}}}+\frac{1}{Q_{\mathrm{sc}}}\right)^{-1},
$$

where $Q_{\mathrm{f}}$ takes into account all other acoustic loss mechanisms, intrinsic and extrinsic, associated with the PMN-PT film. Assuming $Q_{\mathrm{m}}=70$ (Fig. 11(a)) one can readily get $Q_{\mathrm{f}} \approx 103$. This implies that the overall mechanical $Q$-factor of the BAW resonator is limited mainly by the losses inside the PMN-PT film. The rather low $Q$-factor of the PMN-PT film is consistent with the $Q=20$, at $1.3 \mathrm{GHz}$, found for the high quality single-domain PMN-0.33PT film heteroepitaxially grown by magnetron sputtering. ${ }^{17}$ Clear dependences on the diameter of the top electrode (Fig. 11) indicate that one way to increase the $Q$-factor is increasing the resonator lateral size.

\section{SUMMARY}

We present results of characterization and analysis of electroacoustic performance of the intrinsically tunable bulk acoustic wave resonators based on the PMN-PT thin films grown by sol-gel technique. The XRD analysis reveals that the PMN-PT films are strongly [001] textured. The PMN-PT film composition on the morphotropic phase boundary and the [001] orientation provide conditions for the polarization rotation induced by applied dc electric field, which results in enhanced electroacoustic properties of the PMN-PT resonators. The effective electromechanical coupling coefficient of the PMN-PT resonators is up to $13 \%$. Tunability of the series resonance frequency is also rather high, up to $4.0 \%$. It is shown that the electroacoustic performance of the PMN-PT resonators can be described in the frames of the theory of dc field-induced piezoelectric effect in ferroelectrics. Extrinsic acoustic loss in the PMN-PT resonators is analyzed using the model of the wave scattering at reflections from rough interfaces. Mechanical $Q$-factor of the resonators is up to 70 at 4.1 GHz and limited mainly by losses in the PMN-PT film, including the loss associated with generation of shear waves by fringing field at the resonator edges. Clear dependences on the diameter of the top electrode indicate that one way to increase the $Q$-factor is increasing the resonator lateral size.

\section{ACKNOWLEDGMENTS}

This work was partly supported by the projects VRFBAR, CompFBAR (Vetenskapsrådet) and NAFERBio (Vinnova, MVZT), Sweden and Slovenia.

${ }^{1}$ A. Noeth, T. Yamada, P. Muralt, A. K. Tagantsev, and N. Setter, IEEE Trans. Ultrason. Ferroelectr. Freq. Control 57, 379 (2010).

${ }^{2}$ S. A. Sis, V. Lee, J. D. Phillips, and A. Mortazawi, IEEE MTT-S Int. Microwave Symp. Dig. (2012), p. 1.

${ }^{3}$ G. N. Saddik, J. Son, S. Stemmer, and R. A. York, J. Appl. Phys. 109, 091606 (2011).

${ }^{4}$ A. Volatier, E. Defay, M. Aid, A. N'hari, P. Ancey, and B. Dubus, Appl. Phys. Lett. 92, 032906 (2008).

${ }^{5}$ B. Ivira, A. Reinhardt, E. Defay, and M. Aid, in IEEE International Frequency Control Symposium (2008), p. 254.

${ }^{6}$ A. Vorobiev and S. Gevorgian, Appl. Phys. Lett. 96, 212904 (2010).

${ }^{7}$ A. Vorobiev, S. Gevorgian, N. Martirosyan, M. Löffler, and E. Olsson, Appl. Phys. Lett. 101, 232903 (2012).

${ }^{8}$ A. A. Bokov and Z.-G. Yea, J. Appl. Phys. 91, 6656 (2002).

${ }^{9}$ B. Noheda, D. E. Cox, G. Shirane, J. Gao, and Z.-G. Ye, Phys. Rev. B 66, 054104 (2002).

${ }^{10}$ D. Zekria and A. M. Glazer, J. Appl. Cryst. 37, 143 (2004).

${ }^{11}$ I. A. Ivan, M. Rakotondrabe, J. Agnus, R. Bourquin, N. Chaillet, P. Lutz, J.-C. Poncot, R. Duffait, and O. Bauer, Rev. Adv. Mater. Sci. 24, 1 (2010).

${ }^{12}$ P.-C. Wang, H.-S. Luo, X.-M. Pan, D.-L. Li, and Z.-W. Yin, in Proceedings of the 12th IEEE International Symposium on Applications of Ferroelectrics (2001), Vol. 2, p. 537.

${ }^{13}$ R. Skulski, P. Wawrzala, K. Cwikiel, and D. Bochenek, J. Intell. Mater. Syst. Struct. 18, 1049 (2007).

${ }^{14}$ S. O. Leontsev and R. E. Eitel, J. Am. Ceram. Soc. 92, 2957 (2009).

${ }^{15}$ M. Frank, K. S. Moon, and S. Kassegne, Smart Mater. Struct. 19, 035015 (2010).

${ }^{16}$ K. Zhang, S.-H. Choy, L. Zhao, H. Luo, H. L.-W. Chan, and Y. Wang, Microelectron. Eng. 88, 1028 (2011).

${ }^{17}$ K. Wasa, S. Ito, K. Nakamura, T. Matsunaga, I. Kanno, T. Suzuki, H. Okino, T. Yamamoto, S. H. Seo, and D. Y. Noh, Appl. Phys. Lett. 88, 122903 (2006).

${ }^{18}$ A. Vorobiev, S. Gevorgian, M. Löffler, and E. Olsson, J. Appl. Phys. 110, 054102 (2011).

${ }^{19}$ W. Jo, T.-H. Kim, D.-Y. Kim, and S. K. Pabi, J. Appl. Phys. 102, 074116 (2007).

${ }^{20}$ P. Padmini, T. R. Taylor, M. J. Lefevre, A. S. Nagra, R. A. York, and J. S. Speck, Appl. Phys. Lett. 75, 3186 (1999).

${ }^{21}$ J. Hlinka and P. Marton, Phys. Rev. B 74, 104104 (2006).

${ }^{22}$ S. Gevorgian, A. Tagantsev, and A. Vorobiev, Tuneable Film Bulk Acoustic Wave Resonators (Springer, 2013), p. 123.

${ }^{23}$ A. Noeth, T. Yamada, V. O. Sherman, P. Muralt, A. K. Tagantsev, and N. Setter, J. Appl. Phys. 102, 114110 (2007).

${ }^{24}$ J. Berge and S. Gevorgian, IEEE Trans. Ultrason. Ferroelectr. Freq. Control 58, 2768 (2011).

${ }^{25}$ J. Berge, M. Norling, A. Vorobiev, and S. Gevorgian, J. Appl. Phys. 103, 064508 (2008). 
${ }^{26}$ A. Noeth, T. Yamada, V. O. Sherman, P. Muralt, A. K. Tagantsev, and N. Setter, IEEE Trans. Ultrason. Ferr. Freq. Control 54, 2487 (2007).

${ }^{27}$ A. Kvasov and A. K. Tagantsev, J. Appl. Phys. 113, 204104 (2013).

${ }^{28}$ A. Vorobiev and S. Gevorgian, Int. J. Microwave Wireless Technol. 5, 361 (2013)

${ }^{29}$ J. Berge, A. Vorobiev, W. Steichen, and S. Gevorgian, IEEE Microwave Wireless Comp. Lett. 17, 655 (2007).

${ }^{30} \mathrm{~S}$. Gevorgian and A. Vorobiev, in Proceedings of the 40th European Microwave Conference (2010), Vol. 1, p. 1210.

${ }^{31}$ S. Gevorgian, A. Tagantsev, and A. Vorobiev, Tuneable Film Bulk Acoustic Wave Resonators (Springer, 2013), p. 187.
${ }^{32}$ A. Vorobiev and S. Gevorgian, IEEE Trans. Ultrason. Ferr. Freq. Control 61, 840 (2014).

${ }^{33}$ J. Kaitila, RF Bulk Acoustic Wave Filters for Communications, edited by K.-Y. Hashimoto (Artech House, Norwood, MA, 2009), pp. 78-79.

${ }^{34}$ A. Vorobiev, J. Berge, S. Gevorgian, M. Löffler, and E. Olsson, J. Appl, Phys. 110, 024116 (2011).

${ }^{35}$ G. Fattinger and S. Marksteiner, RF Bulk Acoustic Wave Filters for Communications, edited by K.-Y. Hashimoto (Artech House, Norwood, MA, 2009), p. 214.

${ }^{36}$ S. G. Alekseev, G. D. Mansfel'd, N. I. Polzikova, and I. M. Kotelyanskii, Acoust. Phys. 53, 465 (2007). 